\title{
Coupled dynamics in gluon mass generation and the impact of the three-gluon vertex
}

\author{
Daniele Binosi $^{1}$ and Joannis Papavassiliou ${ }^{2}$ \\ ${ }^{1}$ European Centre for Theoretical Studies in Nuclear Physics and Related Areas (ECT*) and Fondazione \\ Bruno Kessler, Villa Tambosi, Strada delle Tabarelle 286, I-38123 Villazzano (Trento), Italy \\ ${ }^{2}$ Department of Theoretical Physics and IFIC, University of Valencia and CSIC, E-46100 Valencia, Spain
}

(Received 2 October 2017; published 26 March 2018)

\begin{abstract}
We present a detailed study of the subtle interplay transpiring at the level of two integral equations that are instrumental for the dynamical generation of a gluon mass in pure Yang-Mills theories. The main novelty is the joint treatment of the Schwinger-Dyson equation governing the infrared behavior of the gluon propagator and of the integral equation that controls the formation of massless bound-state excitations, whose inclusion is instrumental for obtaining massive solutions from the former equation. The self-consistency of the entire approach imposes the requirement of using a single value for the gauge coupling entering in the two key equations; its fulfilment depends crucially on the details of the threegluon vertex, which contributes to both of them, but with different weight. In particular, the characteristic suppression of this vertex at intermediate and low energies enables the convergence of the iteration procedure to a single gauge coupling, whose value is reasonably close to that extracted from related lattice simulations.
\end{abstract}

DOI: 10.1103/PhysRevD.97.054029

\section{INTRODUCTION}

The nonperturbative aspects of the gluon propagator, $\Delta_{\mu \nu}^{a b}(q)$, are considered to be especially relevant for the qualitative and quantitative understanding of a wide range of important physical phenomena, such as confinement, chiral symmetry breaking, and bound-state formation. A particularly interesting feature, which manifests itself both in the Landau gauge and away from it, is the saturation of its scalar form factor, $\Delta\left(q^{2}\right)$, in the deep infrared (IR), i.e., $\Delta(0)=c_{0}>0$. This special behavior was firmly established in a variety of $\mathrm{SU}(2)$ [1-3] and $\mathrm{SU}(3)$ [4-8] largevolume lattice simulations, and has been extensively studied in the continuum within diverse theoretical frameworks [9-41]. In fact, if one were to adopt the notions and terminology put forth in $[17,42]$, the property $\Delta(0)=c_{0}>0$ is a particular case of a more general phenomenon, denominated "gapping." Specifically, the generation of a fundamental mass gap in the Yang-Mills theory manifests itself at the level of the gluon propagator by enforcing the property $\Delta(0)<\infty$. In particular, the so-called "scaling solutions," for which $\Delta(0)=0$, form part of this wide class of "gapped" gluon propagators.

Published by the American Physical Society under the terms of the Creative Commons Attribution 4.0 International license. Further distribution of this work must maintain attribution to the author(s) and the published article's title, journal citation, and DOI. Funded by SCOAP ${ }^{3}$.
One particular framework that has been developed for addressing the complicated dynamics associated with the IR finite gluon propagator arose from the merging of the pinch-technique (PT) [9,43-47] with the background-field method (BFM) [48], to be referred to as "PT-BFM scheme" $[15,49,50]$. Inherent to this scheme is the distinction between background $(B)$ and quantum $(Q)$ gluons, and the proliferation of the possible Green's functions that one may form with them. Particularly relevant for what follows is the distinction between the $Q Q$ and $Q B$ gluon selfenergies, and the $Q^{3}$ and $B Q^{2}$ three-gluon vertices, to be denoted by $\Gamma$ and $\tilde{\Gamma}$, respectively.

We call the reader's attention on two subtle issues related with the PT-BFM framework. First, the Schwinger-Dyson equations (SDEs) that control the evolution of the gluon propagators of the type $Q B$ and $B B$ within this framework display certain desirable truncation properties, which, by virtue of a set of exact identities, denominated backgroundquantum identities (BQIs) [see Eq. (2.1)], are eventually transferred to the conventional $Q Q$ propagator (the one simulated on the lattice). Second, the vertices of the type $\Gamma$ obey the conventional (nonlinear) Slavnov-Taylor identities (STIs), whereas those of the type $\tilde{\Gamma}$ satisfy linear (Abelian) STIs when contracted with the momentum carried by the $B$ field (this momentum coincides with the momentum $q$ entering into the gluon SDE, see below).

Returning to the main problem, as has been shown is a series of earlier contributions to this subject [51-54], in 
order to obtain an IR finite solution out of the aforementioned SDEs one must introduce certain crucial assumptions. Specifically, an indispensable ingredient is the presence of massless poles of the type $1 / q^{2}$ in the vertices with one $B$ leg, which enter into the $Q B$ gluon self-energy. In fact, this particular non-analytic terms must contribute nontrivially to the realization of the STIs satisfied by the corresponding fully-dressed vertices. For instance, in the case of the three-gluon vertex $\tilde{\Gamma}$ employed both in [51-54] and in the present work, a term of the form $\left(q_{\mu} / q^{2}\right)$ $\tilde{C}_{\alpha \beta}(q, r, p)$ must be included in it, which, after the contraction by $q^{\mu}$ and the resulting cancellation of the pole, furnishes a piece of the corresponding STI. We will employ the term "longitudinally coupled" to describe such poles.

The scenario outlined above constitutes a special (non-Abelian) version of the well-known Schwinger mechanism for gauge-boson mass generation [55-60]. The origin of the poles is dynamical, owing to the formation of colored bound-state excitations, which are massless due to the strong binding induced by the YangMills interactions. The integral equations that govern their formation constitute a system of homogeneous linear Bethe-Salpeter equations (BSEs), which determines the derivatives of the corresponding "bound-state wave functions." In the present work, we will simplify the degree of complexity by restricting the possibility of pole formation only in $\tilde{\Gamma}$, thus reducing the aforementioned system into a single BSE, which determines the corresponding derivative, to be denoted by $\tilde{C}_{1}^{\prime}\left(k^{2}\right)$. Note, in fact, that $\tilde{C}_{1}^{\prime}\left(k^{2}\right)$ and the $\tilde{C}_{\alpha \beta}(q, r, p)$ introduced above are intimately connected: $\tilde{C}_{1}^{\prime}\left(k^{2}\right)$ is a special partial derivative of the $g_{\alpha \beta}$ form factor of $\tilde{C}_{\alpha \beta}$ [see Eq. (2.15)].

Evidently, the self-consistent implementation of the dynamical picture described above hinges on the subtle interplay between the BSE and SDE, and the compatibility of the various field-theoretic ingredients that enter in them. The purpose of the present work is to focus on a particularly pivotal aspect of this interplay, and elucidate the decisive impact not only of $\tilde{\Gamma}$, whose $1 / q^{2}$ pole enforces the desired IR finiteness of $\Delta\left(q^{2}\right)$, but especially of $\Gamma$, whose IR structure affects both the kernel of the BSE and a crucial two-loop component of the SDE.

In order to appreciate how the inclusion of $\Gamma$ helps in obtaining a self-consistent picture, let us note that both the SDE and the BSE depend on the value of the strong coupling $\alpha_{s}=g^{2} / 4 \pi$. After renormalization has been implemented, the value of $\alpha_{s}$, fixed at a given subtraction point $\mu$, must be common in all parts of the calculations. In fact, it is a standard text-book statement that the validity of the STIs requires precisely the imposition of such a common value for $\alpha_{s}$. Thus, turning to the case at hand, if we denote the value of $\alpha_{s}$ used in the SDE by $\alpha_{s}^{\mathrm{SDE}}$, and the corresponding value of $\alpha_{s}$ by $\alpha_{s}^{\mathrm{BSE}}$, a priori the relation
$\alpha_{s}^{\mathrm{SDE}}=\alpha_{s}^{\mathrm{BSE}}$ must be fulfilled. However, it turns out that, when the tree-level expression of $\Gamma$ is used in the evaluation of the SDE and the BSE, ${ }^{1}$ the resulting values for $\alpha_{s}^{\mathrm{SDE}}$ and $\alpha_{s}^{\mathrm{BSE}}$ do not coincide. This discrepancy suggests that certain important pieces in the kernels of the SDE and the BSE have been left out, in an unbalanced way; as a result, one is forced to compensate this mismatch by assigning distinct values to $\alpha_{s}^{\mathrm{SDE}}$ and $\alpha_{s}^{\mathrm{BSE}}$.

The main result of the present study is that the nonperturbative behavior of the $Q^{3}$ vertex $\Gamma$ becomes relevant when trying to restore the crucial equality $\alpha_{s}^{\mathrm{SDE}}=\alpha_{s}^{\mathrm{BSE}}$. Note in particular that (a) $\Gamma$ enters linearly in the SDEderived expression that determines the value of $\Delta^{-1}(0)$ and quadratically in the kernel of the BSE, rendering it renormalization group invariant (RGI), and (b) below $1 \mathrm{GeV}$ the vertex $\Gamma$ is suppressed with respect to its tree-level value, reversing its sign around $100 \mathrm{MeV}$, and finally diverging logarithmically at the origin.

If one employs a standard nonperturbative ansatz for $\Gamma$, which encodes the features mentioned in (b), one finds that, indeed, $\alpha_{s}^{\mathrm{SDE}}=\alpha_{s}^{\mathrm{BSE}}$. The common value is given by $\alpha_{s}=0.45$, when the momentum subtraction (MOM) renormalization is implemented at $\mu=4.3 \mathrm{GeV}$. This particular value for $\alpha_{s}$ is to be contrasted with the one obtained (for the same $\mu$ ) from the lattice simulation of the three-gluon vertex $\Gamma$ in [61], namely $\alpha_{s}=0.32$. This discrepancy appears to be more than acceptable given the approximations implemented when deriving both the SDE and the BSE, and, in particular, the simplifications applied in the renormalization of the former, and the truncations imposed when constructing the kernel of the latter.

\section{SCHWINGER MECHANISM AND VERTICES WITH MASSLESS POLES}

Throughout this work, we consider a SU(3) pure YangMills theory (no dynamical quarks). In the Landau gauge, the gluon propagator $\Delta_{\mu \nu}^{a b}(q)=\delta^{a b} \Delta_{\mu \nu}(q)$ has the form

$$
\Delta_{\mu \nu}(q)=-i \Delta\left(q^{2}\right) P_{\mu \nu}(q) ; \quad P_{\mu \nu}(q)=g_{\mu \nu}-\frac{q_{\mu} q_{\nu}}{q^{2}},
$$

\footnotetext{
${ }^{1}$ We emphasize that the use of a tree-level expression for $\Gamma$ does not interfere with one's ability to obtain an IR finite $\Delta\left(q^{2}\right)$; indeed, it is the " $q$-channel" poles, included in $\tilde{\Gamma}$, that are crucial for this purpose. Instead, none of the three quantum legs of $\Gamma$ are irrigated by $q$, given that $\Gamma$ appears "nested" inside the relevant diagrams (this may be clearly seen in Fig. 2). The poles of $\Gamma$ are thus displaced, being of the form $1 / \ell^{2}$, where $\ell$ is one of the virtual loop momenta that are being integrated over. In any case, in the Landau gauge, the "longitudinally coupled" poles of $\Gamma$ are annihilated (both in the SDE and the BSE), because each leg is contracted by the corresponding projection tensor.
} 


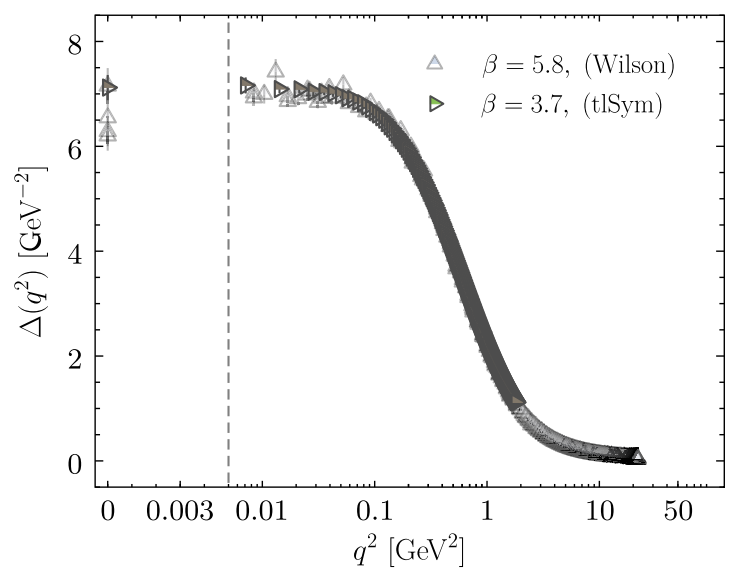

FIG. 1. Lattice data for the quenched Landau gauge gluon propagator obtained from a tree-level Symanzik (tlSym) improved gauge action [61,62] (calibrated following the procedure described in [63]), compared with the corresponding data obtained from a Wilson gauge action [5]. The momentum axis is linear on the left of the vertical dashed line and logarithmic on the right, an artifice that clearly exposes the existence of a saturation point at IR momenta.

where $\Delta\left(q^{2}\right)$ is related to the form factor of the gluon self-energy $\Pi_{\mu \nu}(q)=P_{\mu \nu}(q) \Pi\left(q^{2}\right)$, through $\Delta^{-1}\left(q^{2}\right)=$ $q^{2}+i \Pi\left(q^{2}\right)$. Lattice data for this (quenched) quantity, renormalized at $\mu=4.3 \mathrm{GeV}$, are shown in Fig. 1 and will serve as the main input in the ensuing analysis. In addition, the ghost propagator $D^{a b}\left(q^{2}\right)=i \delta^{a b} D\left(q^{2}\right)$ furnishes the dressing function, $F\left(q^{2}\right)$, defined as $F\left(q^{2}\right)=$ $q^{2} D\left(q^{2}\right)$; in the Landau gauge (again at $\mu=4.3 \mathrm{GeV}$ ), $F(0) \approx 2.9$.

In the PT-BFM framework, the SDE of $\Delta\left(q^{2}\right)$ is expressed in terms of the $Q B$ self-energy $\tilde{\Pi}_{\mu \nu}(q)$, namely (see Fig. 2),

$$
\Delta^{-1}\left(q^{2}\right) P_{\mu \nu}(q)=\frac{q^{2} P_{\mu \nu}(q)+i \tilde{\Pi}_{\mu \nu}(q)}{1+G\left(q^{2}\right)}
$$

where $G\left(q^{2}\right)$ is the $g_{\mu \nu}$ component of a special two-point function [64]. In the Landau gauge only, the important relation $1+G(0)=F^{-1}(0)$ holds exactly $[23,65]$.

The main advantage of expressing the gluon SDE in terms of $\tilde{\Pi}_{\mu \nu}(q)$ rather than $\Pi_{\mu \nu}(q)$ arises from the fact that, when contracted from the side of the $B$-gluon, each fully dressed vertex satisfies a linear (Abelian-like) SlavnovTaylor identity (STI). In particular, when contracted by the momentum carried by the background leg, ${ }^{2}$ the $B Q^{2}$ vertex $\tilde{\Gamma}_{\mu \alpha \beta}$ satisfies (color omitted)

\footnotetext{
${ }^{2}$ Two additional (nonlinear) STIs are fulfilled when contracting with respect to the two quantum legs $\left(r^{\alpha}\right.$ and $\left.p^{\beta}\right)$, but their form is not relevant for the present analysis.
}

$$
q^{\mu} \tilde{\Gamma}_{\mu \alpha \beta}(q, r, p)=i \Delta_{\alpha \beta}^{-1}(r)-i \Delta_{\alpha \beta}^{-1}(p) .
$$

This particular STI furnishes nontrivial constraints on the structure of $\tilde{\Gamma}_{\mu \alpha \beta}(q, r, p)$, but leaves completely undetermined its "transverse" component, $\tilde{\Gamma}_{\mu \alpha \beta}^{\mathrm{T}}(q, r, p)$, which is identically conserved, namely

$q^{\mu} \tilde{\Gamma}_{\mu \alpha \beta}^{\mathrm{T}}(q, r, p)=r^{\alpha} \tilde{\Gamma}_{\mu \alpha \beta}^{\mathrm{T}}(q, r, p)=p^{\beta} \tilde{\Gamma}_{\mu \alpha \beta}^{\mathrm{T}}(q, r, p)=0$.

We now concentrate on the IR dynamics described by the gluon SDE of Eq. (2.2), when combined with the STI of Eq. (2.3), and in conjunction with some crucial assumptions regarding the analytic structure of $\tilde{\Gamma}_{\mu \alpha \beta}(q, r, p)$. In particular, we will briefly review the consequences of the absence/presence of a "longitudinally coupled" pole, with the characteristics mentioned in the Introduction.

To that end, let us first assume that all form factors composing $\tilde{\Gamma}_{\mu \alpha \beta}(q, r, p)$ (i.e., both the "transverse" and the "STI-saturating" components) are well-behaved analytic functions in the limit $q \rightarrow 0$. Then, employing a tensorial basis that does not introduce spurious IR divergences, one may show that the term $\tilde{\Gamma}_{\mu \alpha \beta}^{\mathrm{T}}(q, r, p)$, when inserted into the right-hand side (rhs) of the SDE, gives no contribution to $\Delta^{-1}(0)$.

The determination of the contribution to $\Delta^{-1}(0)$ stemming from the part of $\tilde{\Gamma}_{\mu \alpha \beta}(q, r, p)$ that can be reconstructed from Eq. (2.2) is technically more subtle [53], involving a delicate interplay between the Ward-Takahashi identity (WTI), satisfied by $\tilde{\Gamma}_{\mu \alpha \beta}(q, r, p)$ as $q \rightarrow 0$, and an integral relation known as the "seagull identity" $[53,66]$.

To see this, consider the limit of the STI (2.3) as $q \rightarrow 0$. Then, given that (by assumption) we are dealing with wellbehaved functions, the Taylor expansion of both sides may be carried out. This, in turn, generates the corresponding WTI,

$$
\tilde{\Gamma}_{\mu \alpha \beta}(0, r,-r)=-i \frac{\partial}{\partial r^{\mu}} \Delta_{\alpha \beta}^{-1}(r)
$$

which, when used in the evaluation of the gluon SDE, yields

$$
\Delta^{-1}(0)=\underbrace{\int_{k} \frac{\partial}{\partial k_{\mu}} \mathcal{F}_{\mu}(k)=0}_{\text {seagull identity }} ; \quad \mathcal{F}_{\mu}(k)=k_{\mu} \mathcal{F}\left(k^{2}\right)
$$

where $\mathcal{F}\left(k^{2}\right)=\Delta\left(k^{2}\right)\left[c_{1}+c_{2} Y\left(k^{2}\right)\right]$, with $c_{1}, c_{2} \neq 0$, and (see Fig. 2) 


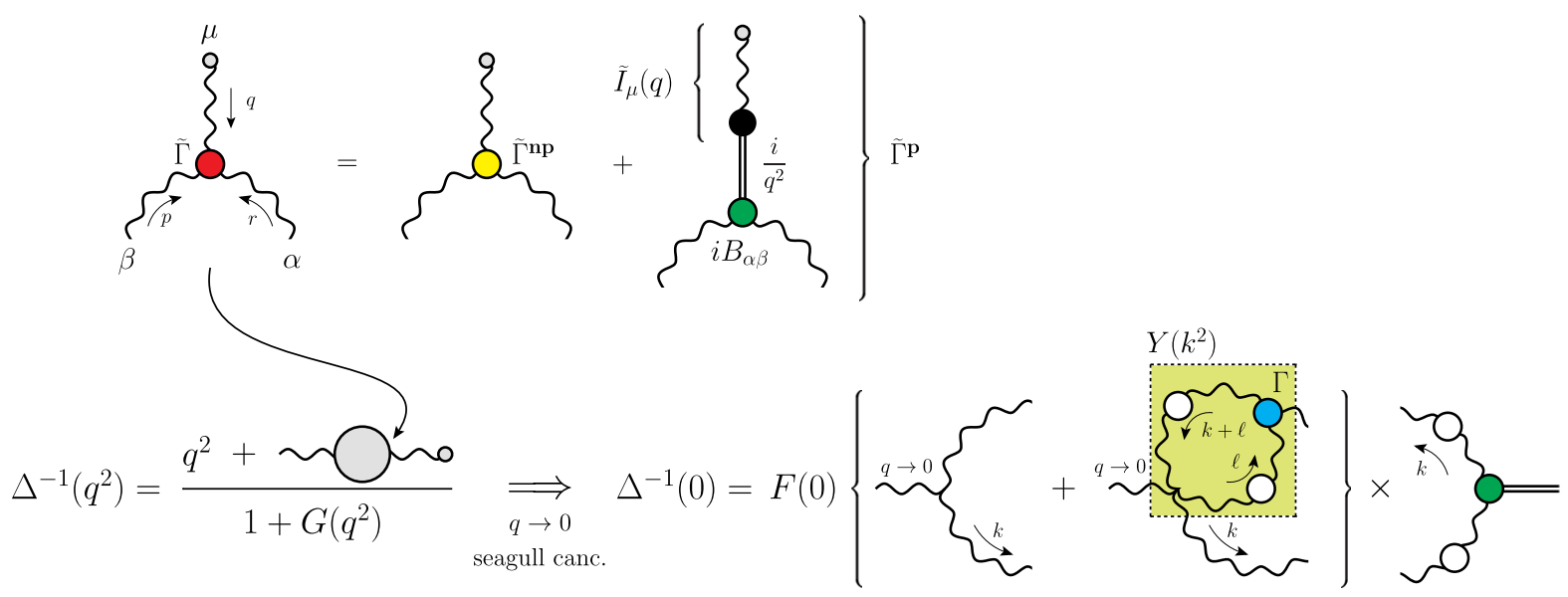

FIG. 2. The procedure leading to the dynamical gluon mass generation within the PT-BFM framework. Notice in particular that since the transition amplitude $\tilde{I}_{\mu}(q)$ has one free index and depends on a single momentum, it is automatically longitudinally coupled: $\tilde{I}_{\mu}(q)=q_{\mu} \tilde{I}\left(q^{2}\right)[51]$. In addition, we set (see also footnote 3 ) $\tilde{C}_{\alpha \beta}=\tilde{I} B_{\alpha \beta}$.

$Y\left(k^{2}\right)=\frac{1}{(d-1)} \frac{k_{\alpha}}{k^{2}} \int_{\ell} \Delta^{\alpha \rho}(\ell) \Delta^{\beta \sigma}(\ell+k) \Gamma_{\sigma \rho \beta}(-\ell-k, \ell, k)$.

Note that we have introduced the dimensional regularization integral measure $\int_{k} \equiv \frac{\mu^{e}}{(2 \pi)^{d}} \int \mathrm{d}^{d} k$, with $d=4-\epsilon$ the space-time dimension, and $\mu$ the 't Hooft mass scale. Thus, we conclude that, under the crucial assumptions regarding the structure of $\tilde{\Gamma}$ mentioned above, one cannot obtain an IR finite solution from the gluon SDE of Eq. (2.2).

In order to circumvent the result of Eq. (2.6), one must allow $\tilde{\Gamma}_{\mu \alpha \beta}$ to contain some type of nonanalytic contributions. In what follows, we will focus our interest on a particular version of this possibility, known in the literature as the Schwinger mechanism $[55,56]$, widely employed for the dynamical generation of a gauge boson mass [57-60].

Specifically, we will assume that the underlying dynamics will give rise to a very special nonanalytic term to $\tilde{\Gamma}$, which contributes nontrivially to the realization of the STI given in Eq. (2.3). In other words, this particular term cannot be written exclusively in terms of the transverse basis, but has a nonvanishing projection in the part that saturates the STI.

In particular, we have

$$
\tilde{\Gamma}_{\mu \alpha \beta}(q, r, p)=\tilde{\Gamma}_{\mu \alpha \beta}^{\mathrm{np}}(q, r, p)+\tilde{\Gamma}_{\mu \alpha \beta}^{\mathbf{p}}(q, r, p),
$$

with

$$
\tilde{\Gamma}_{\mu \alpha \beta}^{\mathbf{p}}(q, r, p)=\frac{q_{\mu}}{q^{2}} \tilde{C}_{\alpha \beta}(q, r, p),
$$

where the superscripts "np" and "p" indicate the "no-pole" and "pole" parts, respectively, and $\tilde{C}_{\alpha \beta}$ is the aforementioned bound-state wave function. Its most general Lorentz decomposition is given by ${ }^{3}$

$$
\begin{aligned}
\tilde{C}_{\alpha \beta}(q, r, p)= & \tilde{C}_{1} g_{\alpha \beta}+\tilde{C}_{2} p_{\alpha} p_{\beta}+\tilde{C}_{3} r_{\alpha} r_{\beta}+\tilde{C}_{4} r_{\alpha} p_{\beta} \\
& +\tilde{C}_{5} p_{\alpha} r_{\beta},
\end{aligned}
$$

where $\tilde{C}_{i}=\tilde{C}_{i}(q, r, p)$. Notice that the Bose-symmetry of the vertex under the exchange $(\alpha, r) \leftrightarrow(\beta, p)$ imposes the relation $\tilde{C}_{\alpha \beta}(0, r,-r)=0$, which implies in particular that

$$
\tilde{C}_{1}(0, r,-r)=0 \text {. }
$$

As has been explained in detail in [51,54], if we work in the Landau the only relevant form factor in the above tensorial decomposition is $\tilde{C}_{1}$.

Next, in order to preserve the BRST symmetry of the theory, we demand that all STIs maintain their exact form in the presence of these poles; therefore, Eq. (2.3) will now read

$q^{\mu} \tilde{\Gamma}_{\mu \alpha \beta}^{\mathrm{np}}(q, r, p)+\tilde{C}_{\alpha \beta}(q, r, p)=i \Delta_{\alpha \beta}^{-1}(r)-i \Delta_{\alpha \beta}^{-1}(p)$.

Taking the limit of Eq. (2.12) as $q \rightarrow 0$ and matching the lowest order terms in $q$, the corresponding WTI becomes

\footnotetext{
${ }^{3}$ In $[51,54]$ the "transition amplitude" $\tilde{I}\left(q^{2}\right)$, was explicitly factored out (see Fig. 2), and the corresponding form factors were denoted by $B_{i}(q, r, p)$. In particular, the form factors associated with $g_{\alpha \beta}$ in the two bases are related by $\tilde{C}_{1}=\tilde{I}\left(q^{2}\right) B_{1}$.
} 


$$
\begin{aligned}
\tilde{\Gamma}_{\mu \alpha \beta}^{\mathrm{np}}(0, r,-r)= & -i \frac{\partial}{\partial r^{\mu}} \Delta_{\alpha \beta}^{-1}(r) \\
& -\left\{\frac{\partial}{\partial q^{\mu}} \tilde{C}_{\alpha \beta}(q, r,-r-q)\right\}_{q=0} .
\end{aligned}
$$

The presence of the second term on the rhs of Eq. (2.13) has far-reaching consequences for the IR behavior of $\Delta$. Specifically, a repetition of the steps leading to Eq. (2.6) reveals that, whereas the first term on the rhs of Eq. (2.13) reproduces again Eq. (2.6) (and its contribution thus vanishes), the second term survives, giving

$$
\begin{aligned}
\Delta^{-1}(0)= & \frac{3}{2} g^{2} C_{A} F(0) \int_{k} k^{2} \Delta^{2}\left(k^{2}\right)\left[1-\frac{3}{2} g^{2} C_{A} Y\left(k^{2}\right)\right] \\
& \times \tilde{C}_{1}^{\prime}\left(k^{2}\right),
\end{aligned}
$$

where

$$
\tilde{C}_{1}^{\prime}\left(k^{2}\right)=\lim _{q \rightarrow 0}\left\{\frac{\partial \tilde{C}_{1}(q, k,-k-q)}{\partial(k+q)^{2}}\right\},
$$

and $C_{A}$ is the Casimir eigenvalue of the adjoint representation $[N$ for $\mathrm{SU}(N)]$. Note that the one- and two-loop dressed contributions enter into the mass condition (2.14) with a different relative sign, a fact that is crucial for the ensuing analysis.

We conclude this section with some pertinent remarks.

(1) We emphasize that in the above demonstration we have assumed that the transverse parts of the vertex do not contain non-bound-state poles, namely poles that would be there even if the Schwinger mechanism had been switched off. It is important to maintain a sharp distinction regarding the nature of the poles, because, depending on the tensorial basis employed, the $\tilde{C}_{\alpha \beta}(q, r, p)$ given in Eq. (2.10) may contribute (in part) to the corresponding transverse part; for instance, if one re-expresses $\tilde{C}_{\alpha \beta}(q, r, p)$ in the Ball-Chiu (BC) basis [67], ${ }^{4}$ with the exception of the first term, all others contribute to $\tilde{\Gamma}_{\mathrm{BC}}^{\mathrm{T}}$. Evidently, such contributions to $\tilde{\Gamma}^{\mathrm{T}}$ would be proportional to the form factors $\tilde{C}_{i}(q, r, p)$, being the result of simply rewriting $\tilde{C}_{\alpha \beta}(q, r, p)$ in another basis. However, if poles of different origin were allowed in $\tilde{\Gamma}^{\mathrm{T}}$, accompanied by new form factors, say $\tilde{Y}_{i}(q, r, p)$, they would in general interfere with the above construction. It is relatively straightforward to establish, for instance, in the $\mathrm{BC}$ basis, that if the $\tilde{Y}_{i}(q, r, p)$ are of the form $\tilde{Y}_{i}(q, r, p)=$ $c_{i}(q, r, p) / q^{2}$, and are substituted into the gluon

\footnotetext{
${ }^{4}$ We use the tensorial structures denoted by $t_{i}(i=1,2,3,4)$ in Eq. (4.6) of [68].
}

SDE, then they too contribute to the final value of $\Delta^{-1}(0)$.

(2) Related to the previous point, we are not aware of any lattice or SDE statements regarding the analytic structure of the transverse parts of the three-gluon vertex, and in particular the possibility of them developing a massless pole. In particular, and mainly due to kinematic reasons, present lattice simulations cannot confirm or discard such structures. For instance, the typical quantity considered in lattice simulation of the three-gluon vertex (Landau gauge) is the ratio $[69,70]$

$$
R(q, r, p)=\frac{\mathcal{N}(q, r, p)}{\mathcal{D}(q, r, p)},
$$

where

$$
\begin{aligned}
\mathcal{N}(q, r, p)= & \Gamma_{\alpha \mu \nu}^{(0)}(q, r, p) P^{\alpha \rho}(q) P^{\mu \sigma}(r) P^{\nu \tau}(p) \\
& \times \Gamma_{\rho \sigma \tau}(q, r, p), \\
\mathcal{D}(q, r, p)= & \Gamma_{\alpha \mu \nu}^{(0)}(q, r, p) P^{\alpha \rho}(q) P^{\mu \sigma}(r) P^{\nu \tau}(p) \\
& \times \Gamma_{\rho \sigma \tau}^{(0)}(q, r, p) .
\end{aligned}
$$

First of all, due to its special Lorentz structure, the term $\tilde{\Gamma}_{\mu \alpha \beta}^{\mathbf{p}}(q, r, p)$ in Eq. (2.9) automatically drops out from $R$. Moreover, we have checked using $\tilde{\Gamma}_{\mathrm{BC}}^{\mathrm{T}}$, that the resulting kinematic prefactors cancel completely the pole assumed to be contained in $\tilde{Y}_{i}(q, r, p)$, giving, at most, a finite contribution (for $i=4$ ), which is mixed with those of other (regular) form factors, and could not be possibly disentangled from the data.

Similarly, at the level of the SDE that controls the vertex $\tilde{\Gamma}$, one may expect that diagrams containing (IR finite) gluon propagators will furnish results that are well behaved as $q \rightarrow 0$. The type of diagrams that are known to produce nonanalytic structures are the "triangle" graphs containing a ghost loop; this is so because the three ghost propagators entering there are nonperturbatively massless, namely $D\left(k^{2}\right)=$ $F\left(k^{2}\right) / k^{2}$, with $F\left(k^{2}\right)$ finite. However, the only type of divergence stemming from them, which has been analytically identified [42,71-77], and observed on the lattice $[61,62,69,70,78]$, is logarithmic (see next section). Thus, even though no rigorous proof may be offered at this level, we consider the aforementioned scenario of the transverse parts containing primary poles rather remote.

(3) Note that the derivation of Eq. (2.14) involves the Taylor expansion of $\tilde{C}_{1}(q, k,-k-q)$ around $q=0$. Due to the validity of Eq. (2.11), the first term in this expansion is linear in $q$, and its co-factor is precisely the $\tilde{C}_{1}^{\prime}\left(k^{2}\right)$, defined in Eq. (2.15). It is clear that 
$\tilde{C}_{1}^{\prime}\left(k^{2}\right)$ should not vanish identically, because, in such a case, $\Delta^{-1}(0)=0$; this is precisely what we will establish in the next section, by solving the BSE that controls $\tilde{C}_{1}^{\prime}\left(k^{2}\right)$. Evidently, the nonvanishing of $\tilde{C}_{1}^{\prime}\left(k^{2}\right)$ means that the pole $1 / q^{2}$ multiplying $\tilde{C}_{1}(q, k,-k-q)$ does not get washed out. In other words, $\tilde{C}_{1}(0, k,-k)$ vanishes linearly in $q$, and even though it makes the total divergence weaker, does not eliminate it (that would have been the case if the Taylor expansion of $\tilde{C}_{1}(q, k,-k-q)$ would start with the second instead of the first derivative). The persistence of the divergence may be clearly seen in the three-dimensional plot of the quantity $\tilde{C}_{1}(q, r, p) / q^{2}$, shown in the second panel of Fig. 6.

(4) We emphasize that if one wants to obtain an infrared finite gluon propagator, which is the purpose of this exercise, the "divergence" introduced in $\tilde{\Gamma}_{\mu \alpha \beta}^{\mathrm{p}}(q, r, p)$ must be precisely that of a pole. This is so because, with the additional properties of the form factor $\tilde{C}_{1}$ mentioned above, $\tilde{\Gamma}_{\mu \alpha \beta}^{\mathbf{p}}(q, r, p)$ is the only way to reproduce, at the level of the gluon SDE, exactly the required structure $\left(g_{\mu \nu}-q_{\mu} q_{\nu} / q^{2}\right) \Delta^{-1}\left(q^{2}\right)$, with the finite $\Delta^{-1}(0)$, given by Eq. (2.14). If, instead, any other power of the form $q^{-2(1+b)}$, with $b \neq 0$, were to be introduced, one would arrive at a structure $\left(g_{\mu \nu}-q_{\mu} q_{\nu} / q^{2}\right) \Delta_{b}^{-1}\left(q^{2}\right)$, with $\Delta_{b}^{-1}\left(q^{2}\right)=q^{-2 b} \Delta^{-1}$ $\left(q^{2}\right)$. Then, in the limit $q \rightarrow 0$, if $b>0$ we obtain a gluon propagator that vanishes at the origin, while for $b<0$ a divergent one; evidently, both possibilities contradict the lattice results mentioned in the Introduction.

(5) The above point is also relevant for the proper interpretation of the BSE results of the next section. In particular, the derivation of the BSE for $\tilde{C}_{1}^{\prime}\left(k^{2}\right)$ relies on the matching of the most divergent contribution on both sides of this equation. It is relatively straightforward to establish that one would arrive at the same equation for $\tilde{C}_{1}^{\prime}\left(k^{2}\right)$ even if the power of the "divergence" were different than a pole (but sufficiently strong to suppress other contributions). Thus, when studied in isolation, the nonvanishing of the $\tilde{C}_{1}^{\prime}\left(k^{2}\right)$ does not necessarily prove the formation of a genuine pole. However, the BSE is not to be understood in isolation, but rather coupled to the corresponding gluon SDE. Then, in the light of the previous point, the nonvanishing $\tilde{C}_{1}^{\prime}\left(k^{2}\right)$ may be indeed interpreted as the BSE amplitude for the generation of a massless pole.

\section{BSE FOR THE MASSLESS BOUND-STATES}

The dynamical equation that governs $\tilde{C}_{1}\left(k^{2}\right)$ may be derived from the SDE satisfied by $\tilde{\Gamma}_{\mu \alpha \beta}(q, r, p)$, as $q \rightarrow 0$. In this limit, the derivative term becomes the leading contribution, given that $\tilde{C}_{\alpha \beta}(0, r,-r)=0$, and the resulting homogeneous equation assumes the form of a BSE (see Fig. 3), given by [54]

$$
\begin{aligned}
f^{a m n} \lim _{q \rightarrow 0} \tilde{C}_{\alpha \beta}(q, r, p)= & f^{a b c} \lim _{q \rightarrow 0}\left\{\int_{k} \tilde{C}_{\gamma \delta}(q, k,-k-q) \Delta^{\gamma \rho}(k)\right. \\
& \left.\times \Delta^{\delta \sigma}(k+q) \mathcal{K}_{\rho \alpha \beta \sigma}^{b m n c}(-k, r, p, k+q)\right\} .
\end{aligned}
$$

To proceed further, we will approximate the four-gluon BS kernel $\mathcal{K}$ by the lowest-order set of diagrams appearing in its skeleton expansion, given by the diagrams $\left(b_{1}\right),\left(b_{2}\right)$, and $\left(b_{3}\right)$, shown in the second line of Fig. 3. It turns out

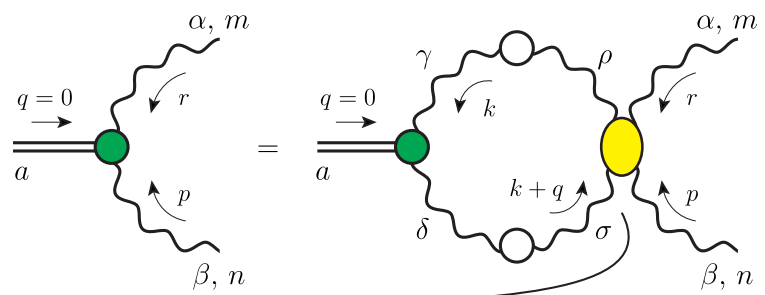

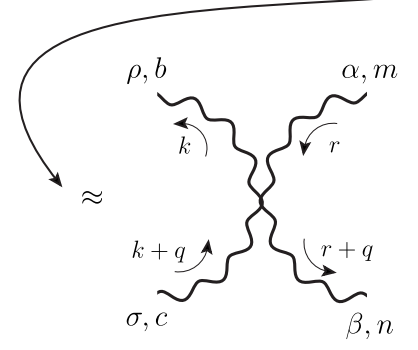

$\left(b_{1}\right)$

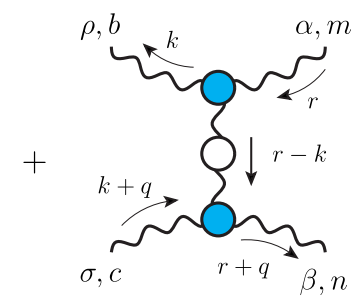

$\left(b_{2}\right)$

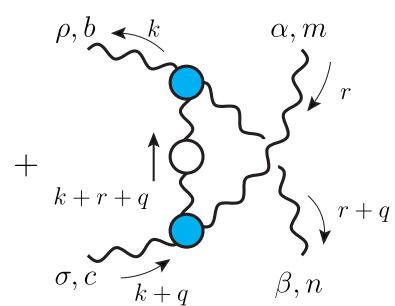

$\left(b_{3}\right)$

FIG. 3. The BSE satisfied by the bound-state wave function $\tilde{C}_{\alpha \beta}$ (upper line) and the simplified four-gluon kernel used. 
that, if we use the tree-level four-gluon vertex in the evaluation of $\left(b_{1}\right)$, its contribution in the above kinematic limit vanishes. Diagrams $\left(b_{2}\right)$ and $\left(b_{3}\right)$, which carry a statistical factor of $1 / 2$, are considered to contain fully dressed gluon propagators and three gluon vertices $\Gamma$ (note that all gluons are of the $Q$-type). As a consequence, the resulting BSE does not depend on the value of the MOM subtraction point $\mu$, because the two graphs composing its kernel may be written as the "square" of the formally RGI combination [by virtue of Eq. (6.2)]

$\mathcal{R}^{\mu \alpha \beta}\left(k_{1}, k_{2}, k_{3}\right)=g \Delta\left(k_{1}\right) \Delta^{1 / 2}\left(k_{2}\right) \Gamma^{\mu \alpha \beta}\left(k_{1}, k_{2}, k_{3}\right)$,

namely, setting $q=0,\left(b_{2}\right) \sim \mathcal{R}(-k, k-r, r) \mathcal{R}(k, r-k,-r)$ and $\left(b_{3}\right) \sim \mathcal{R}(-k, k+r,-r) \mathcal{R}(k,-r-k, r)$.

The vertex $\Gamma$ contains 14 form factors [67], whose nonperturbative structure, albeit subject of various studies [42,71-77], is only partially known. Therefore, for the purposes of the present work, we will consider the simple ansatz,

$$
\Gamma_{\mu \alpha \beta}\left(k_{1}, k_{2}, k_{3}\right)=f\left(k_{2}\right) \Gamma_{\mu \alpha \beta}^{(0)}\left(k_{1}, k_{2}, k_{3}\right),
$$

where $\Gamma^{(0)}$ is the standard tree-level expression of the vertex, and the form factor $f(r)$ is considered to be a function of a single kinematic variable. We emphasize (see also footnote in page 4) that, unlike $\tilde{\Gamma}$, the $\Gamma_{\mu \alpha \beta}\left(k_{1}, k_{2}, k_{3}\right)$ does not contain poles in $1 / q^{2}$, bur rather in $1 / k_{i}^{2}$ $(i=1,2,3)$; such poles are integrated over and are not important for obtaining the desired gluon propagator. In fact, $\Gamma_{\mu \alpha \beta}\left(k_{1}, k_{2}, k_{3}\right)$ is contracted by three transverse projection operators, ${ }^{5}$ which completely annihilate any such contribution. For that reason, the ansatz proposed in Eq. (3.3) is compatible with the rest of our construction.

Then, using Eq. (3.3) into Eq. (3.1), we arrive at the final equation,

$$
\begin{aligned}
\tilde{C}_{1}^{\prime}\left(r^{2}\right)= & \frac{8 \pi}{3} \alpha_{s} C_{A} \int_{k} \tilde{C}_{1}^{\prime}\left(k^{2}\right) \frac{(r \cdot k)\left[r^{2} k^{2}-(r \cdot k)^{2}\right]}{r^{4} k^{2}(k+r)^{2}} \\
& \times \Delta^{2}(k) \Delta(k+r) f^{2}(k+r) \\
& \times\left[8 r^{2} k^{2}+6(r \cdot k)\left(r^{2}+k^{2}\right)\right. \\
& \left.+3\left(r^{4}+k^{4}\right)+(r \cdot k)^{2}\right] .
\end{aligned}
$$

The functional form we will employ for $f(k+r)$ is motivated by a considerable number of lattice simulations and studies in the continuum. In particular, for certain characteristic kinematic configurations (such as the symmetric and the soft gluon limits), the vertex is suppressed

\footnotetext{
${ }^{5}$ Note that, in addition to the projection operators due to the internal Landau gauge propagators, the derivation of the BSE involves a contraction by $P_{\alpha \beta}(r)$, in order to project out the $\tilde{C}_{1}$ component of $\tilde{C}_{\alpha \beta}$.
}

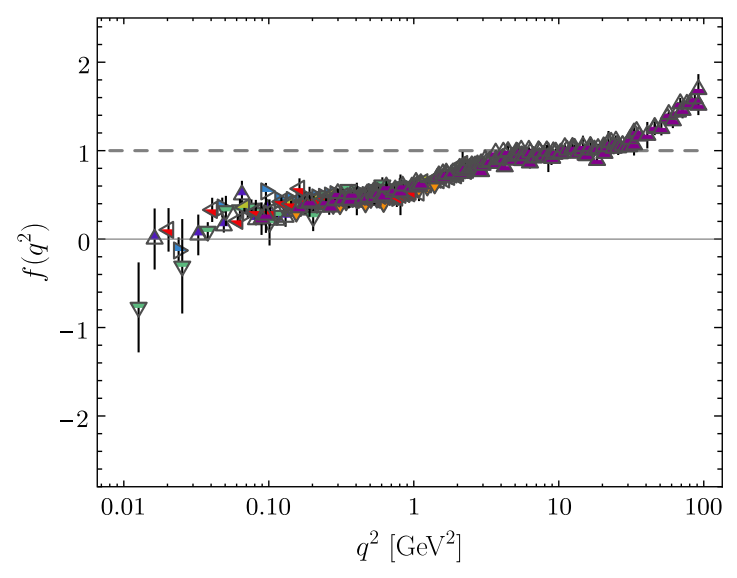

FIG. 4. Compilation of SU(3) lattice data (evaluated with various $\beta$, volumes and actions) for the form factor $f$ in the symmetric configuration $[61,62]$.

with respect to its tree-level value, reverses its sign for relatively small momenta (an effect known as "zero crossing"), and finally diverges at the origin [42,71-77]. The reason for this particular behavior may be traced back to the delicate balance between contributions originating from gluon loops, which are "protected" by the corresponding gluon mass, and the "unprotected" logarithms coming from the ghost loops that contain massless ghosts. Early lattice indication for a zero crossing in SU(2) Yang-Mills theories can be found in $[69,70]$, whereas the effect has been recently confirmed to be present also in the case of $\mathrm{SU}(3)$ theories [61,62,78]. A compilation of the lattice data of $[61,62]$, properly normalized by dividing out the coupling $[g=2$ at $\mu=4.3 \mathrm{GeV}$ for the set at hand, corresponding to $\alpha_{s}=0.32$ ], is shown in Fig. 4 .

We end this section by briefly discussing the numerical procedure followed for solving Eq. (3.4). Introducing spherical coordinates, this equation can be rewritten schematically as

$$
\tilde{C}_{1}^{\prime}(x)=\alpha_{s} \int_{0}^{\pi} \mathrm{d} \theta \int_{0}^{b} \mathrm{~d} y \mathcal{K}(x, y, \theta) \tilde{C}_{1}^{\prime}(y),
$$

where one should have $b=\infty$ but in practice is limited to a finite $b \gg 1$. By expanding the unknown function in terms of Chebishev polynomials of the first kind,

$$
\tilde{C}_{1}^{\prime}(x)=\frac{c_{0}}{2}+\sum_{k=1}^{n} c_{k} T_{k}(x),
$$

we then map the problem into determining the $n+1$ coefficients characterizing this expansion $[79,80]$. To do that, we discretize the variable $x \in[0, b]$ as

$$
x_{j}=\frac{b}{2} \cos \left(\frac{\pi}{n}(n-j)\right)+\frac{b}{2}, \quad j=0, \ldots, n ;
$$


that is, the $x_{j}$ correspond to the extrema of the $n$th Chebishev polynomial in this interval.

It is then relatively easy to prove that finding solutions to our original equation is equivalent to identifying the values of $\alpha_{s}$ for which the matrix $A-\alpha_{s} B$ is singular, where

$$
\begin{aligned}
A_{i j} & =\delta T_{i}\left(\frac{2 x_{j}-b}{b}\right) \\
B_{i j} & =\delta \int_{0}^{\pi} \mathrm{d} \theta \int_{0}^{b} \mathrm{~d} y \mathcal{K}\left(x_{j}, y, \theta\right) T_{i}\left(\frac{2 x_{j}-b}{b}\right),
\end{aligned}
$$

and $\delta=1 / 2$ if $i=0$, and $\delta=1$ otherwise. Provided that such $\alpha_{s}$ exists, one can then determine all the expansion coefficients $c_{k}$ by simply setting to $c_{0}=1$ and then solving the resulting reduced eigenvector equation.

\section{RUNNING GLUON MASS FROM THE BSE}

The upshot of the construction presented so far is that the Schwinger mechanism endows the gluon with a mass, which is the physical way of interpreting the saturation of the gluon propagator at the origin. In this section, we will extend this notion to the case of the running gluon mass, which dates back to the pioneering work of [9], ${ }^{6}$ and outline the procedure that permits its reconstruction from the solution $\tilde{C}_{1}^{\prime}\left(r^{2}\right)$ obtained from the BSE.

In the absence of poles, the validity of Eq. (2.6) suggests that $\Delta^{-1}\left(q^{2}\right)=q^{2} J\left(q^{2}\right)$, where the function $J\left(q^{2}\right)$ captures the perturbative contributions and diverges as $\ln q^{2}$ at the origin. Instead, the IR saturation of $\Delta^{-1}\left(q^{2}\right)$ motivates the physical parametrization,

$$
\Delta^{-1}\left(q^{2}\right)=q^{2} J\left(q^{2}\right)+m^{2}\left(q^{2}\right),
$$

with $m^{2}(0) \neq 0$. Note that $J\left(q^{2}\right)$ is also affected by the presence of the mass, since the perturbative logarithms of the form $\ln q^{2}$ that stem from gluon loops are "protected" by the mass, being (qualitatively) replaced by $\ln \left(q^{2}+m^{2}\left(q^{2}\right)\right)$.

It is important to emphasize that the decomposition introduced in Eq. (4.1) is mathematically not unique, because, in the absence of any additional criterion, parts of the $q^{2} J\left(q^{2}\right)$ term may be reassigned to $m^{2}\left(q^{2}\right)$. This is to be contrasted with what happens in the corresponding decomposition of the inverse quark propagator according to $S^{-1}(p)=A\left(p^{2}\right) \not p-B\left(p^{2}\right)$, where the different Dirac nature of the two terms makes their identification mathematically unambiguous.

Notwithstanding this limitation, a meaningful definition of $m^{2}\left(q^{2}\right)$ may be reached through the judicious allotment of the terms $\tilde{\Gamma}_{\mu \alpha \beta}^{\mathrm{np}}(q, r, p)$ and $\tilde{\Gamma}_{\mu \alpha \beta}^{\mathbf{p}}(q, r, p)$ entering in the

\footnotetext{
${ }^{6}$ For a detailed treatise of the meaning and origins of this mass, its role in confinement and screening, its nontrivial interplay with the gauge-field condensates, and its usefulness in describing QCD phenomenology, the reader is referred to [81].
}

STI of Eq. (2.12). Specifically, after introducing Eq. (4.1) in the rhs of Eq. (2.12), it is natural to associate the $J$ terms with the $q^{\mu} \tilde{\Gamma}_{\mu \alpha \beta}^{\text {np }}$ on the lhs and, correspondingly,

$$
\tilde{C}_{\alpha \beta}(q, r, p)=m^{2}\left(p^{2}\right) P_{\alpha \beta}(p)-m^{2}\left(r^{2}\right) P_{\alpha \beta}(r) .
$$

Focusing on the $g_{\alpha \beta}$ components of Eq. (4.2), we obtain

$$
\tilde{C}_{1}(q, r, p)=m^{2}\left(p^{2}\right)-m^{2}\left(r^{2}\right),
$$

which, in the limit $q \rightarrow 0$, leads to the important result [51]

$$
\tilde{C}_{1}^{\prime}\left(r^{2}\right)=\frac{\mathrm{d} m^{2}\left(r^{2}\right)}{\mathrm{d} r^{2}} .
$$

Then, upon integration,

$$
m^{2}(x)=\Delta^{-1}(0)+\int_{0}^{x} \mathrm{~d} y \tilde{C}_{1}^{\prime}(y),
$$

where $x=q^{2}$ and $y=r^{2}$. Eq. (4.5) establishes thus a possible link between the solution of the BSE (3.4) and what has been identified in the literature with the dynamically generated gluon mass [82]. However, in order for the quantity $m^{2}\left(q^{2}\right)$ to admit a running mass interpretation in the sense familiar from the quark case, it needs to: (i) be a monotonically decreasing function of $q^{2}$; (ii) vanish in the UV, i.e., satisfy $m^{2}(\infty)=0$.

To explore the implications of these requirements, let $S^{\prime}$ be a general solution of the BSE (3.4) corresponding to a certain (eigen)value of the strong coupling, $\alpha_{s}=\alpha_{s}^{\mathrm{BSE}}$. The typical shape of such solutions is shown in Fig. 5. Then, one has $\tilde{C}_{1}^{\prime}(x)=c S^{\prime}(x)$, where $c$ is a normalization constant that needs to be determined. To this end, observe that, with the kernel used, $S^{\prime}$ is positive definite; then, the requirement of a monotonically decreasing $m^{2}(x)$ forces $c$ to be negative: $c=-|c|$. Furthermore, the condition $m^{2}(\infty)=0$ fixes its modulus, since Eq. (4.5) implies

$$
\Delta^{-1}(0)=|c| \int_{0}^{\infty} \mathrm{d} y S^{\prime}(y),
$$

with $|c|=0.0076$ for the solution shown in Fig. 5 . Substitution of Eq. (4.6) into Eq. (4.5) yields

$$
m^{2}(x)=|c| \int_{x}^{\infty} \mathrm{d} y S^{\prime}(y),
$$

which, upon integration, gives rise to the squared running mass shown in Fig. 5; it may be accurately fitted by

$$
m^{2}\left(q^{2}\right)=m^{2}(0) /\left[1+\left(q^{2} / m_{1}^{2}\right)^{1+p}\right],
$$

with $m_{1}=0.36 \mathrm{GeV}$ and $p=0.1$, in excellent agreement with the behavior found in [83]. 

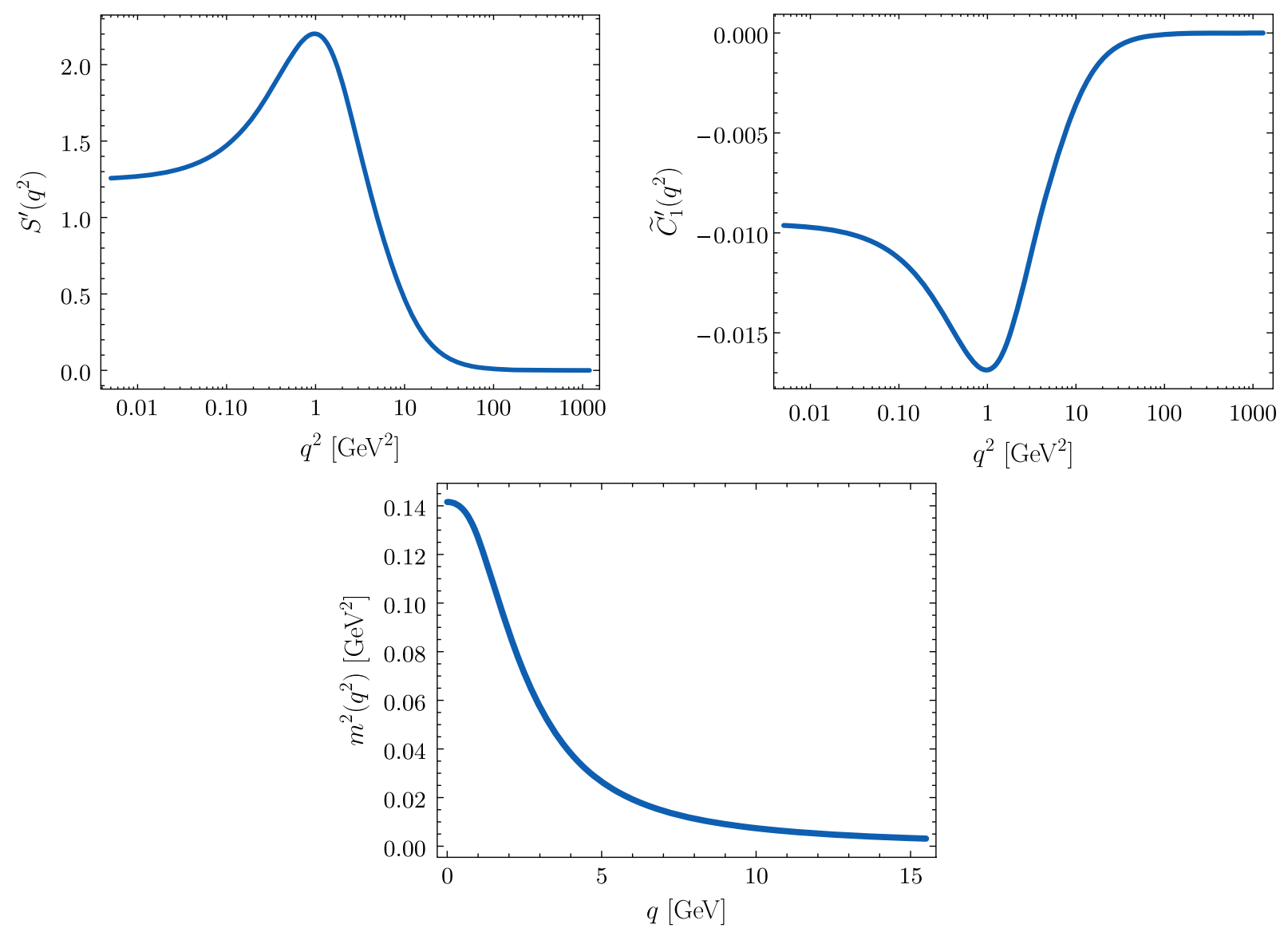

FIG. 5. From the left-top clockwise: A typical solution of the BSE (3.4), the corresponding normalized solution, and the associated dynamically generated gluon mass.

We finally note that the special ansatz introduced in Eq. (4.3) for $\tilde{C}_{1}(q, r, p)$ allows its full reconstruction, once $\mathrm{m}^{2}\left(q^{2}\right)$ is obtained. In particular, using the functional form for $m^{2}\left(q^{2}\right)$ shown in the second panel of Fig. 5, evaluated for the arguments $r^{2}$ and $(q+r)^{2}$, we obtain the threedimensional curve shown in the left panel of Fig. 6 for the value $\theta=0$ being $\theta$ the angle between $q$ and $r$. When dividing this quantity by $x$ one sees the developing of a

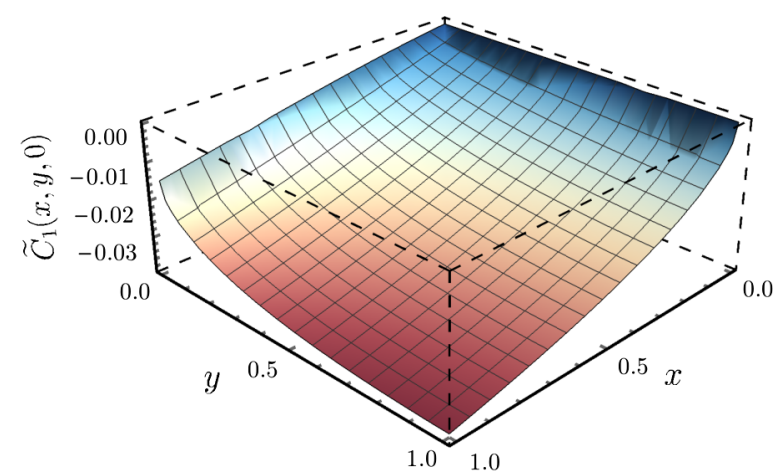

massless pole as $x \rightarrow 0$ which is ultimately responsible for the generation of the gluon mass.

\section{BSE-SDE consistency through the inclusion of $\boldsymbol{\Gamma}_{\mu \alpha \beta}$}

Having introduced all necessary ingredients pertinent to the problem, in this section we present the main novel results of the present study.

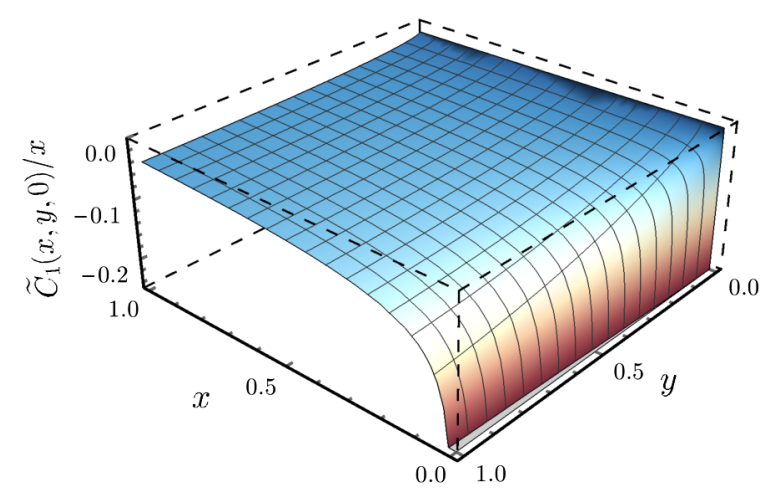

FIG. 6. The form factor $\tilde{C}_{1}(x, y, \theta)(\theta$ being the angle between $q$ and $r)$ reconstructed from the special ansatz Eq. (4.3) using the mass obtained in Eq. (4.7). Left panel: $\tilde{C}_{1}$ evaluated at $\theta=0$. Right panel: The same quantity as before but now divided by $x$, and therefore showing the presence of a pole in the $x \rightarrow 0$ limit. In both case the units for $x$ and $y$ are $\mathrm{GeV}^{2}$. 
Specifically, as mentioned in the Introduction, a selfconsistent treatment of both the SDE and the BSE would require that the $\alpha_{s}$ entering in them be the same, given that its value has been fixed within a given renormalization scheme (MOM) and at a given scale $\mu$. If, instead, different values for $\alpha_{s}$ need be employed in these two integral equations in order to obtain compatible dynamics, this is an indication that certain important pieces have been left out, due to the unavoidable truncations implemented to them. As we will see in this section, this discrepancy, which is indeed there, may be eliminated by including appropriate physically motivated nonperturbative corrections to the vertex $\Gamma_{\mu \alpha \beta}$, which enters in the graphs of both the SDE and the BSE. We remind the reader that $\Gamma$ plays a completely different role than $\tilde{\Gamma}$ in this context: with a tree-level $\tilde{\Gamma}$ we cannot obtain a IR finite gluon propagator; with a tree-level $\Gamma$ we simply get the values of $\alpha_{s}$ off (see also footnote in page 4).

Let us now return to Eq. (2.14), whose derivation was carried out before renormalization. Its renormalization may be carried out by introducing the standard renormalization constants for the propagators, vertices, and the coupling. Then, using the constraints that the various STIs impose on these constants, all quantities entering into Eq. (2.14) can be converted into renormalizaed ones, and the replacement

$$
1-\frac{3}{2} g^{2} C_{A} Y\left(k^{2}\right) \rightarrow Z_{3}-\frac{3}{2} Z_{4} g_{R}^{2} C_{A} Y_{R}\left(k^{2}\right)
$$

must be implemented on its rhs, with $Z_{3}$ and $Z_{4}$ the renormalization constants of the $Q^{3}$ and $Q^{4}$ vertices, respectively.

The presence of $Z_{3}$ and $Z_{4}$ converts the computation of the rhs of Eq. (2.14) into a highly nontrivial exercise, which requires, among other things, the detailed knowledge of the structure of the $Q^{3}$ and $Q^{4}$ vertices. Therefore, as is common in this type of analysis, we will simplify the situation by setting $Z_{3}=Z_{4}=1$.

At this point we will couple the SDE with the BSE. The way we do this is by substituting Eq. (4.6) (which is obtained from the BSE) on the left-hand side of the SDE in Eq. (2.14). The resulting equality may be written as a second-order algebraic equation for $\alpha_{s}$, given by

$$
A \alpha_{s}^{2}+B \alpha_{s}+C=0
$$

where, passing to Euclidean space and using spherical coordinates,

$$
\begin{aligned}
& A=\frac{3 C_{A}^{2}}{32 \pi^{3}} F(0) \int_{0}^{\infty} \mathrm{d} y y^{2} \Delta^{2}(y) Y(y) S^{\prime}(y), \\
& B=-\frac{3 C_{A}}{8 \pi} F(0) \int_{0}^{\infty} \mathrm{d} y y^{2} \Delta^{2}(y) S^{\prime}(y), \\
& C=-\int_{0}^{\infty} \mathrm{d} y S^{\prime}(y) .
\end{aligned}
$$

with $A>0$ and $B, C<0$. The unique positive solution of Eq. (5.2) is given by

$$
\alpha_{s}^{\mathrm{SDE}}=\frac{-B+\sqrt{B^{2}-4 A C}}{2 A},
$$

which shows how the existence of a positive coupling relies on a delicate interplay between the strength of the one- and two-loop dressed contributions in the gluon SDE.

We will now perform a numerical analysis in order to establish if the equality $\alpha_{s}^{\mathrm{SDE}}=\alpha_{s}^{\mathrm{BSE}}$ can be indeed realized, and, if so, at what value of the strong coupling $\alpha_{s}$.

In order to fully appreciate the importance of employing a nontrivial $f$ in this context, let us set $f=1$ both in Eqs. (2.7) and (3.4). Then, a straightforward calculation yields the rather disparate set of values $\alpha_{s}^{\mathrm{SDE}}=0.42$ and $\alpha_{s}^{\mathrm{BSE}}=0.27$. As we will see, the effect of using a physically motivated $f$ will be a slight increase in $\alpha_{s}^{\mathrm{SDE}}$ combined with a considerable increase in $\alpha_{s}^{\mathrm{BSE}}$.

Let us choose for $f$ a fit to the data of Fig. 4, given by [61]

$$
\begin{aligned}
f\left(q^{2}\right)= & \lambda\left[1+b \ln \frac{q^{2}+\mathcal{M}^{2}}{\mu^{2}+\mathcal{M}^{2}}+c \ln \frac{q^{2}}{\mu^{2}}\right. \\
& \left.+e \frac{\mathcal{M}^{2}\left(q^{2}-\mu^{2}\right)}{\left(q^{2}+\mathcal{M}^{2}\right)\left(\mu^{2}+\mathcal{M}^{2}\right)}\right],
\end{aligned}
$$

with $\mu=4.3 \mathrm{GeV}$ the renormalization scale. We set $b=e=-5.30, c=5.40, \mathcal{M}=0.124 \mathrm{GeV}$, but leave the scale factor $\lambda$ undetermined for the moment.

Next, using the same three-gluon vertex approximation (3.3), Eq. (2.7) yields (in spherical coordinates ${ }^{7}$ and $d=4$ )

$$
\begin{aligned}
i Y(y)= & \frac{1}{24 \pi^{3}} \int_{0}^{\infty} \mathrm{d} t t \int_{0}^{\pi} \mathrm{d} \omega \sin ^{4} \omega[5+(y+\sqrt{y t} \cos \omega) / u] \\
& \times f(u) \Delta(t) \Delta(u) .
\end{aligned}
$$

We emphasize that $Y$ is computed for the first time using full gluon propagators and a nonperturbative ansatz for the three-gluon vertex; this is a major improvement, given that all previous treatments of this quantity were purely perturbative (one loop) [82].

We then proceed as follows. To begin with, both in the evaluation of (3.4) and Eq. (5.4) we use as input for $\Delta\left(k^{2}\right)$ and $F(0)$ the lattice data of [5]. Then, we set in Eq. (5.5) the convenient starting value $\lambda_{0}=1$, and determine the value of the coupling $\alpha_{s}^{\mathrm{BSE}}=\alpha_{0}$ for which the BSE (3.4) yields the nontrivial solution $S^{\prime}$; specifically, we find that $\alpha_{0}=0.61$. Next, we substitute $S^{\prime}$ into Eq. (5.3) and compute the coefficients $A_{0}, B_{0}$ and $C_{0}$ of Eq. (5.3), whose values are (all in $\mathrm{GeV}^{2}$ ) $A_{0}=156.2, B_{0}=-40.7$ and $C_{0}=-18.5$. Substituting them into Eq. (5.4), one obtains

\footnotetext{
${ }^{7}$ Here we set $t=\ell^{2}$ and $u=(k+\ell)^{2}$ and $k \cdot \ell=\sqrt{y t} \cos \omega$.
} 


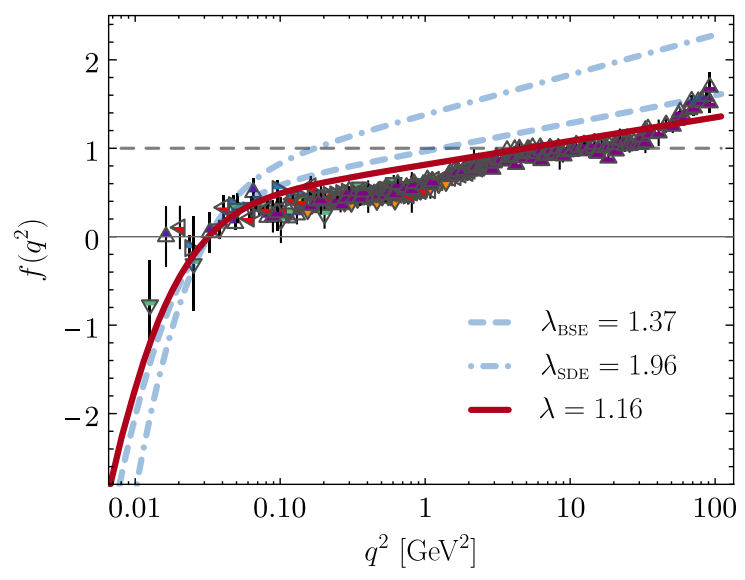

FIG. 7. The three-gluon vertex form factor $f\left(q^{2}\right)$ at $\lambda=1.16$ which leads to the equality $\alpha_{s}^{\mathrm{SDE}}=\alpha_{s}^{\mathrm{BSE}}=0.45$ (red continuous curve). The dashed (light blue) lines show the different form factors needed in the BSE (dashed) and SDE (dot-dashed) to force the equality at $\alpha_{s}^{\mathrm{SDE}}=\alpha_{s}^{\mathrm{BSE}}=0.32$.

$\alpha_{s}^{\mathrm{SDE}}=0.5$; evidently, $\alpha_{s}^{\mathrm{SDE}} \neq \alpha_{0}$. In order to achieve the desired equality $\alpha_{s}^{\mathrm{SDE}}=\alpha_{s}^{\mathrm{BSE}}$, note that, if $\lambda$ is moved from $\lambda_{0}=1$, the BSE will yield precisely the same solution as before provided that its coupling is rescaled to $\alpha_{s}^{\mathrm{BSE}}=$ $\alpha_{0} / \lambda^{2}$ (recall that the BSE is quadratic in $f$ ). In addition, since $Y$ is linear in $f$, we will simply have that $A \rightarrow \lambda A_{0}$, while $B$ and $C$ remain at their initial values. Therefore, imposing the condition $\alpha_{s}^{\mathrm{SDE}}=\alpha_{s}^{\mathrm{BSE}}$ implies that the scale factor $\lambda$ has to be such that

$$
\frac{-B_{0}+\sqrt{B_{0}^{2}-4 \lambda A_{0} C_{0}}}{2 \lambda A_{0}}=\frac{\alpha_{0}}{\lambda^{2}},
$$

or, equivalently,

$$
C_{0} \lambda^{3}+\alpha_{0} B_{0} \lambda+\alpha_{0}^{2} A_{0}=0,
$$

whose only real solution is $\lambda \approx 1.16$. A shown in Fig. 7 , the $f$ obtained from Eq. (5.5) using this special value for $\lambda$ fits particularly well the lattice data. Thus, the two couplings converge to the single value $\alpha_{s}^{\mathrm{BSE}}=\alpha_{s}^{\mathrm{SDE}}=0.45$, corresponding to $g=2.4$ at $\mu^{2}=4.3 \mathrm{GeV}$, which is $20 \%$ off the value used for $g$ in the lattice simulations mentioned above.

As a final possibility, let us assign to the SDE and the BSE different forms of $f$, by setting $\lambda_{\mathrm{BSE}} \neq \lambda_{\mathrm{SDE}}$. This difference may be considered as a simple way of accounting for the fact that, while in the SDE all arguments of $f(x, y, z)$ are integrated over (being virtual), in the BSE the third argument is associated with the external momentum $p$; this, in turn, may modify slightly the corresponding integrated strengths. Then, a straightforward repetition of the iteration procedure described above reveals that one may obtain $\alpha_{s}^{\mathrm{BSE}}=\alpha_{s}^{\mathrm{SDE}}=0.32$ by choosing $\lambda_{\mathrm{BSE}}=1.37$ and $\lambda_{\mathrm{SDE}}=1.96$; the corresponding $f$ are shown in Fig. 7 .

\section{STABILITY UNDER RENORMALIZATION SCALE VARIATIONS}

The analysis presented in the previous section has been carried out at a particular value of the renormalization point, namely $\mu=4.3 \mathrm{GeV}$. Given the importance of the consistency condition between the DSE and BSE couplings, it necessary to discuss its stability under changes of the renormalization point $\mu$.

The Green's functions that affect the consistency condition, namely the gluon propagator, the ghost dressing functions, and the three-gluon vertex, are renormalized as [84]

$$
\begin{aligned}
\Delta\left(q^{2}\right) & =Z_{A}^{-1} \Delta_{0}\left(q^{2}\right) ; \quad F\left(q^{2}\right)=Z_{c}^{-1} F_{0}\left(q^{2}\right) ; \\
\Gamma^{\mu \alpha \beta} & =Z_{3} \Gamma_{0}^{\mu \alpha \beta},
\end{aligned}
$$

where the suffix " 0 " denotes the bare (cutoff-dependent but $\mu$-independent) quantities. In addition, the coupling constant $g$ renormalizes according to

$$
g=Z_{g}^{-1} g_{0} ; \quad Z_{g}=Z_{3} Z_{A}^{-3 / 2} .
$$

At the formal level, changes in the renormalization scale $\mu$ amount to finite renormalizations, implemented by means of the quantities $z_{A}\left(\mu^{2}, \bar{\mu}^{2}\right), z_{c}\left(\mu^{2}, \bar{\mu}^{2}\right)$, and $z_{3}\left(\mu^{2}, \bar{\mu}^{2}\right)$, which take the corresponding function from a reference value $\mu$ to a new value $\bar{\mu}$, according to

$$
\begin{aligned}
& \Delta\left(q^{2}, \bar{\mu}^{2}\right)=z_{A}\left(\bar{\mu}^{2}, \mu^{2}\right) \Delta\left(q^{2}, \mu^{2}\right), \\
& F\left(q^{2}, \bar{\mu}^{2}\right)=z_{c}\left(\bar{\mu}^{2}, \mu^{2} 2\right) F\left(q^{2}, \mu^{2}\right), \\
& f\left(q^{2}, \bar{\mu}^{2}\right)=z_{3}\left(\bar{\mu}^{2}, \mu^{2}\right) f\left(q^{2}, \mu^{2}\right) .
\end{aligned}
$$

In addition, at the reference value of $\mu=4.3 \mathrm{GeV}$, we have that

$$
\alpha_{s}^{\mathrm{BSE}}\left(\mu^{2}\right)=\alpha_{s}^{\mathrm{SDE}}\left(\mu^{2}\right):=\alpha_{s}=0.45,
$$

and (all in $\mathrm{GeV}^{2}$ units)

$A=\lambda A_{0}=181.2 ; \quad B=B_{0}=-40.7$

$C=C_{0}=-18.5$.

In what follows, we will express the condition for the equality of $\alpha_{s}^{\mathrm{BSE}}\left(\bar{\mu}^{2}\right)=\alpha_{s}^{\mathrm{SDE}}\left(\bar{\mu}^{2}\right)$ in terms of these $A, B, C$, $\alpha_{s}$, i.e., the values at the reference scale $\mu$ ), and the corresponding $z_{A}, z_{c}$, and $z_{3}$.

We start with the BSE. As discussed in Sec. III, the BSE is formally RGI, which means that any changes on the renormalization scale implemented on its ingredients $\Delta$ and $f$ must be exactly compensated by a corresponding rescaling in the value of $\alpha_{s}^{\mathrm{BSE}}$. Specifically, with the rescalings described in Eq. (6.3), and using Eq. (6.4), we find 


$$
\alpha_{s}^{\mathrm{BSE}}\left(\bar{\mu}^{2}\right)=z_{A}^{-3} z_{3}^{-2} \alpha_{s} .
$$

Turning to the SDE, notice that, when the ingredients entering into $A$ and $B$ are rescaled according to Eq. (6.3), i.e., renormalized at $\bar{\mu}$, the new quantities, denoted by $\bar{A}$ and $\bar{B}$, are given by [see Eq. (5.3)]

$$
\bar{A}=z_{A}^{4} z_{c} z_{3} A, \quad \bar{B}=z_{A}^{2} z_{c} B,
$$

while the coefficient $C$ remains unchanged, since it is given by the integral of the solution of the BSE $S^{\prime}(y)$, which is $\mu$ independent. Thus, the equivalent of Eq. (5.4) becomes

$$
\alpha_{S}^{\mathrm{SDE}}\left(\bar{\mu}^{2}\right)=\frac{-\bar{B}+\sqrt{\bar{B}^{2}-4 \bar{A} C}}{2 \bar{A}},
$$

and the requirement of equality between couplings, after use of Eq. (6.6), yields

$$
\frac{-\bar{B}+\sqrt{\bar{B}^{2}-4 \bar{A} C}}{2 \bar{A}}=z_{A}^{-3} z_{3}^{-2} \alpha_{s} .
$$

Using then Eq. (6.7), and after elementary algebraic manipulations, we arrive at the following condition

$$
C z_{3}^{3}+z_{3}\left(z_{A}^{-1} z_{c}\right) \alpha_{s} B+\left(z_{A}^{-2} z_{c}\right) \alpha_{s}^{2} A=0 .
$$

Note that in the limiting case where $z_{A}=z_{c}=1$ (that is, we are renormalizing at $\mu=4.3 \mathrm{GeV}$ ), the above equation reduces to that of Eq. (5.8), with the obvious identification $z_{3}=\lambda$. Then, if $A \rightarrow A_{0}$, one finds $z_{3}=\lambda=1.16$; whereas, with $A=\lambda A_{0}$ the equation is trivially satisfied for $z_{3}=1$.

The way to proceed is then the following. We will sample the interval $\bar{\mu} \in[2.4,4.3] \mathrm{GeV}$ with a $0.1 \mathrm{GeV}$ step, and treat $z_{A}$ and $z_{c}$ as known quantities, which can be obtained from the fits of the corresponding lattice data and within the momentum subtraction scheme (MOM) we employ, through the expressions

$$
z_{A}\left(\bar{\mu}^{2}, \mu^{2}\right)=\frac{1}{\bar{\mu}^{2} \Delta\left(\bar{\mu}^{2}, \mu^{2}\right)} ; \quad z_{c}\left(\bar{\mu}^{2}, \mu^{2}\right)=\frac{1}{F\left(\bar{\mu}^{2}, \mu^{2}\right)} .
$$

The values of $z_{A}\left(\bar{\mu}^{2}, \mu^{2}\right)$ and $z_{c}\left(\bar{\mu}^{2}, \mu^{2}\right)$ obtained will be then substituted into Eq. (6.10), which will solved treating $z_{3}$ as an unknown quantity; the values found for $z_{3}$ will then enforce the equality $\alpha_{s}^{\mathrm{BSE}}\left(\bar{\mu}^{2}\right)=\alpha_{s}^{\mathrm{SDE}}\left(\bar{\mu}^{2}\right)$, for the various renormalization scales $\bar{\mu}$. On the left panel of Fig. 8 we show the values of $z_{A}$ and $z_{c}$, given by Eq. (6.11), that we use as input, together with the $z_{3}$ obtained by solving Eq. (6.10).

However, strictly speaking, $z_{3}$ is not an independent quantity; its value can be determined, at least in principle, in the same way as $z_{A}\left(\bar{\mu}^{2}, \mu^{2}\right)$ and $z_{c}\left(\bar{\mu}^{2}, \mu^{2}\right)$, namely from the behavior of the curve $f\left(q^{2}, \mu^{2}\right)$, shown in Fig. 7. Specifically, denoting the corresponding factor by $z_{3}^{f}$, we have,

$$
z_{3}^{f}\left(\bar{\mu}^{2}, \mu^{2}\right)=\frac{1}{f\left(\bar{\mu}^{2}, \mu^{2}\right)} .
$$

The corresponding set of values is shown by the dasheddotted line on the left panel of Fig. 8; even though, ideally, one must have $z_{3}^{f}=z_{3}$, the coincidence between the two curves is rather satisfactory, showing a maximum deviation of only $6 \%$. Given the vast difference between the two procedures used for obtaining $z_{3}$, this near coincidence suggests that the BSE-SDE consistency condition obtained
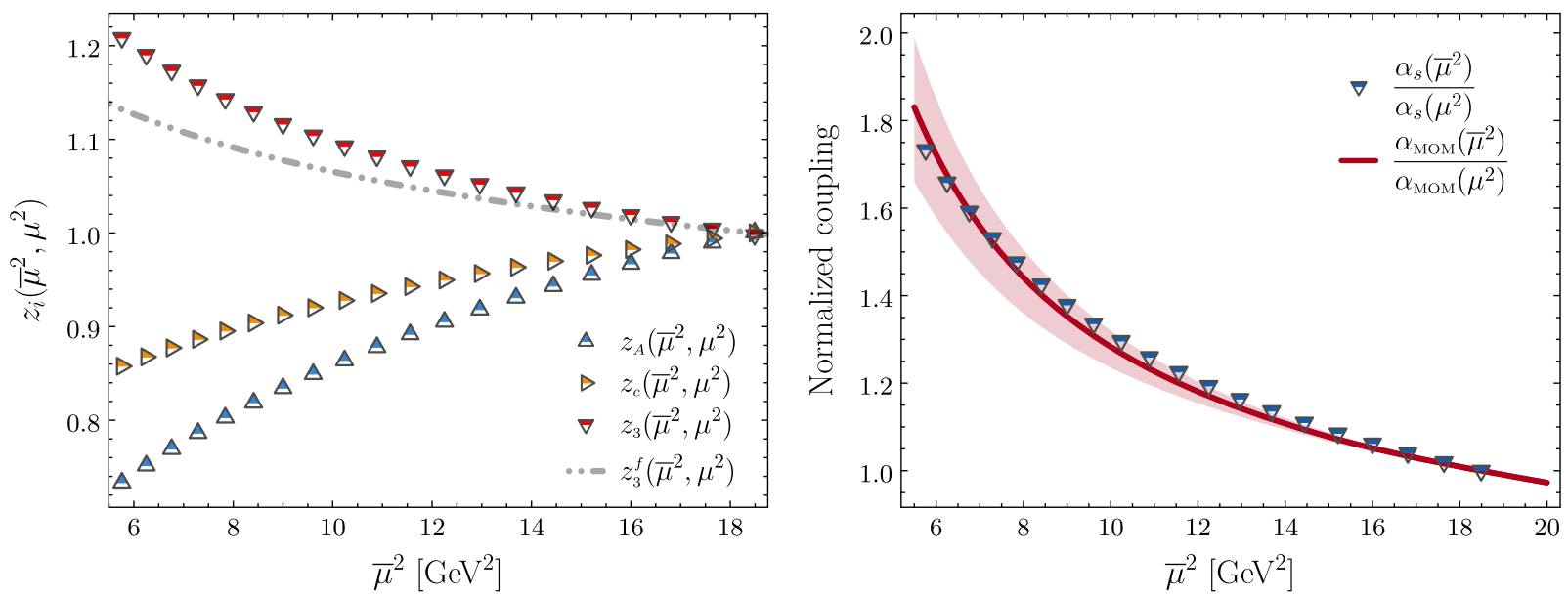

FIG. 8. (Left panel) The renormalization factors $z_{A}, z_{c}$ and $z_{3}$ for various values of the renormalization scale $\bar{\mu}$. (Right panel) The varying of the coupling $\alpha_{s}$ with $\bar{\mu}$ obtained by imposing the consistency condition $\alpha_{s}^{\mathrm{SDE}} \equiv \alpha_{s}^{\mathrm{BSE}}$ (triangles). The curve obtained is then compared with the one predicted by the perturbative running coupling in the MOM scheme, $\alpha_{\mathrm{MOM}}\left(q^{2}\right)$, up to four loops for different values of $\Lambda_{\mathrm{QCD}}$ (see text). 
in the previous section is particularly robust under variations of the renormalization scale.

There is an additional self-consistency check that one may implement, this time at the level of the $\alpha_{s}\left(\bar{\mu}^{2}\right)$. In particular, after obtaining the appropriate set of $z_{3}$, one may use directly Eq. (6.6) to form the ratio $\alpha_{s}\left(\bar{\mu}^{2}\right) / \alpha_{s}\left(\mu^{2}\right)$; the scale-dependence of this ratio is shown on the right panel of Fig. 8 (triangles). This result may in turn be compared with the corresponding ratio $\alpha_{\mathrm{MOM}}\left(\bar{\mu}^{2}\right) / \alpha_{\mathrm{MOM}}\left(\mu^{2}\right)$, which may be reconstructed from the analysis of [85]. The latter ratio is shown by the red curve (obtained when using $\Lambda_{\mathrm{QCD}}=$ $0.41 \mathrm{GeV}$ in the expression derived in [85]), with the band obtained when varying the same parameter within 0.35 and $0.45 \mathrm{GeV}$ (corresponding, respectively, to the lower and upper part of the band). Evidently, even though the starting values of $\alpha_{s}\left(\mu^{2}\right)$ and $\alpha_{\mathrm{MOM}}\left(\mu^{2}\right)$ are different, the good coincidence between the two ratios indicates that the scaledependence of the two couplings is quite similar.

\section{CONCLUSIONS}

We have carried out an extensive analysis of the interlocked dynamics between the SDE of the gluon propagator $\Delta\left(q^{2}\right)$ and a BSE that generates massless bound state poles. These poles constitute an indispensable ingredient of the particular realization of the Schwinger mechanism employed in a series of works in order to obtain IR finite (massive) solutions for $\Delta\left(q^{2}\right)$. The notion of coupling the two equations is novel, and its possible ramifications for the overall self-consistency of the entire formalism have not been explored before in the relevant literature.

Our three main results may be summarized as follows. First, we have obtained a running gluon mass, displaying all expected physical features, directly from the solution of the BSE. This possibility was envisaged in earlier works [52], but the two conditions discussed after Eq. (4.5), which are crucial for obtaining a positive-definite and monotonically decreasing gluon mass, were not fully appreciated. Second, we have carried out a nonperturbative computation of the quantity $Y$, whose role is crucial for obtaining from the SDE a positive-definite gluon mass. Third, we have demonstrated that the inclusion of the three-gluon vertex is of paramount importance for the fulfilment of a basic selfconsistency requirement. In particular, the nontrivial IR dynamics of this vertex compensate the original discrepancy in the value of $\alpha_{s}$ used in the SDE and the BSE sectors, allowing finally for a single common value, $\alpha_{s}=0.45$.

The deviation from the $\alpha_{s}=0.32$ estimated from the lattice simulations of $[61,62]$ may be attributed to a variety of reasons.

To begin with, the skeleton expansion of the BSE kernel has been truncated at the lowest order, shown in Fig. 3 . It would be very important to verify the impact of the next- order corrections ("one-loop" fully dressed). In fact, even the impact of graph $\left(b_{1}\right)$, whose vanishing seems to be an accident of setting the four-gluon vertex at tree level, ought to be reconsidered, using a more complete structure for this vertex $[86,87]$.

In addition, the transition from Eqs. (5.1) to (5.2) was implemented by setting into the former $Z_{3}=Z_{4}=1$. A more complete treatment of this issue has been given in [83]; the resulting kernel, however, is substantially more difficult to calculate, and only Ansätze have been studied thus far. Unfortunately, the complicated nature of this problem makes progress in this direction rather slow.

Turning to $f$, it is clear that the form of Eq. (3.3) is rather restrictive, given that the full tensorial basis for expanding $\Gamma$ consists of 14 elements. In addition, $f$ has been considered to be a function of a single variable (symmetric configuration: $q^{2}=r^{2}=p^{2}$ ). Clearly, a more complete integration over all available momenta and angles could shift the coincidence value of $\alpha_{s}$ closer to $\alpha_{s}=0.32$, as exemplified in the last part of Sec. $\mathrm{V}$ by employing $\lambda \mathrm{BSE} \neq \lambda \mathrm{DSE}$.

Last but not least, the assumption that only the vertex $\tilde{\Gamma}$ develops a massless pole may have to be revisited, allowing the remaining vertices, and especially the ghost-gluon vertex, to form part of a more complex BSE system.

We hope to return to some of the issues mentioned above in the near future.

\section{ACKNOWLEDGMENTS}

We thank J. Rodriguez-Quintero for furnishing the lattice data of Fig. 1. The research of J.P. is supported by the Spanish Ministerio de Educación y Cultura (MEYC) under FPA2014-53631-C2-1-P and SEV-2014-0398, and by Generalitat Valenciana under Grant No. Prometeo II/ 2014/066.
[1] A. Cucchieri and T. Mendes, Proc. Sci., LAT (2007) 297 [arXiv:0710.0412].

[2] A. Cucchieri and T. Mendes, Phys. Rev. Lett. 100, 241601 (2008).

[3] A. Cucchieri and T. Mendes, Phys. Rev. D 81, 016005 (2010).
[4] P. O. Bowman, U. M. Heller, D. B. Leinweber, M. B. Parappilly, A. Sternbeck, L. von Smekal, A. G. Williams, and J. Zhang, Phys. Rev. D 76, 094505 (2007).

[5] I. Bogolubsky, E. Ilgenfritz, M. Muller-Preussker, and A. Sternbeck, Phys. Lett. B 676, 69 (2009). 
[6] O. Oliveira and P. Silva, Proc. Sci., LAT2009 (2009) 226 [arXiv:0910.2897].

[7] A. Ayala, A. Bashir, D. Binosi, M. Cristoforetti, and J. Rodriguez-Quintero, Phys. Rev. D 86, 074512 (2012).

[8] P. Bicudo, D. Binosi, N. Cardoso, O. Oliveira, and P. J. Silva, Phys. Rev. D 92, 114514 (2015).

[9] J. M. Cornwall, Phys. Rev. D 26, 1453 (1982).

[10] M. Lavelle, Phys. Rev. D 44, R26 (1991).

[11] F. Halzen, G. I. Krein, and A. A. Natale, Phys. Rev. D 47, 295 (1993).

[12] O. Philipsen, Nucl. Phys. B628, 167 (2002).

[13] A. P. Szczepaniak and E. S. Swanson, Phys. Rev. D 65, 025012 (2001).

[14] A. C. Aguilar and A. A. Natale, J. High Energy Phys. 08 (2004) 057.

[15] A. C. Aguilar and J. Papavassiliou, J. High Energy Phys. 12 (2006) 012.

[16] K.-I. Kondo, Phys. Rev. D 74, 125003 (2006).

[17] J. Braun, H. Gies, and J. M. Pawlowski, Phys. Lett. B 684, 262 (2010).

[18] D. Epple, H. Reinhardt, W. Schleifenbaum, and A. Szczepaniak, Phys. Rev. D 77, 085007 (2008).

[19] A. C. Aguilar, D. Binosi, and J. Papavassiliou, Phys. Rev. D 78, 025010 (2008).

[20] Ph. Boucaud, J. P. Leroy, A. Le Yaouanc, J. Micheli, O. Pène, and J. Rodríguez-Quintero, J. High Energy Phys. 06 (2008) 099.

[21] D. Dudal, J. A. Gracey, S. P. Sorella, N. Vandersickel, and H. Verschelde, Phys. Rev. D 78, 065047 (2008).

[22] C. S. Fischer, A. Maas, and J. M. Pawlowski, Ann. Phys. (Amsterdam) 324, 2408 (2009).

[23] A. C. Aguilar, D. Binosi, J. Papavassiliou, and J. RodriguezQuintero, Phys. Rev. D 80, 085018 (2009).

[24] J. Rodriguez-Quintero, J. High Energy Phys. 01 (2011) 105.

[25] D. R. Campagnari and H. Reinhardt, Phys. Rev. D 82, 105021 (2010).

[26] M. Tissier and N. Wschebor, Phys. Rev. D 82, 101701 (2010).

[27] K.-I. Kondo, Phys. Rev. D 82, 065024 (2010).

[28] M. Pennington and D. Wilson, Phys. Rev. D 84, 119901 (2011).

[29] P. Watson and H. Reinhardt, Phys. Rev. D 85, 025014 (2012).

[30] K.-I. Kondo, Phys. Rev. D 84, 061702 (2011).

[31] J. Serreau and M. Tissier, Phys. Lett. B 712, 97 (2012).

[32] S. Strauss, C. S. Fischer, and C. Kellermann, Phys. Rev. Lett. 109, 252001 (2012).

[33] I. C. Cloet and C. D. Roberts, Prog. Part. Nucl. Phys. 77, 1 (2014).

[34] F. Siringo, Phys. Rev. D 90, 094021 (2014).

[35] D. Binosi, L. Chang, J. Papavassiliou, and C. D. Roberts, Phys. Lett. B 742, 183 (2015).

[36] A. Aguilar, D. Binosi, and J. Papavassiliou, Phys. Rev. D 91, 085014 (2015).

[37] M. Q. Huber, Phys. Rev. D 91, 085018 (2015).

[38] M. A. L. Capri, D. Dudal, D. Fiorentini, M. S. Guimaraes, I. F. Justo, A. D. Pereira, B. W. Mintz, L. F. Palhares, R. F. Sobreiro, and S. P. Sorella, Phys. Rev. D 92, 045039 (2015).

[39] D. Binosi, C. Mezrag, J. Papavassiliou, C. D. Roberts, and J. Rodriguez-Quintero, Phys. Rev. D 96, 054026 (2017).
[40] S. D. Głazek, M. Gómez-Rocha, J. More, and K. Serafin, Phys. Lett. B 773, 172 (2017).

[41] F. Gao, S.-X. Qin, C. D. Roberts, and J. RodriguezQuintero, Phys. Rev. D 97, 034010 (2018).

[42] A. K. Cyrol, L. Fister, M. Mitter, J. M. Pawlowski, and N. Strodthoff, Phys. Rev. D 94, 054005 (2016).

[43] J. M. Cornwall and J. Papavassiliou, Phys. Rev. D 40, 3474 (1989).

[44] A. Pilaftsis, Nucl. Phys. B487, 467 (1997).

[45] D. Binosi and J. Papavassiliou, Phys. Rev. D 66, 111901(R) (2002).

[46] D. Binosi and J. Papavassiliou, J. Phys. G 30, 203 (2004).

[47] D. Binosi and J. Papavassiliou, Phys. Rep. 479, 1 (2009).

[48] L. F. Abbott, Nucl. Phys. B185, 189 (1981).

[49] D. Binosi and J. Papavassiliou, Phys. Rev. D 77, 061702 (2008).

[50] D. Binosi and J. Papavassiliou, J. High Energy Phys. 11 (2008) 063.

[51] A. Aguilar, D. Ibanez, V. Mathieu, and J. Papavassiliou, Phys. Rev. D 85, 014018 (2012).

[52] D. Ibañez and J. Papavassiliou, Phys. Rev. D 87, 034008 (2013).

[53] A. C. Aguilar, D. Binosi, C. T. Figueiredo, and J. Papavassiliou, Phys. Rev. D 94, 045002 (2016).

[54] A. C. Aguilar, D. Binosi, and J. Papavassiliou, Phys. Rev. D 95, 034017 (2017).

[55] J. S. Schwinger, Phys. Rev. 125, 397 (1962).

[56] J. S. Schwinger, Phys. Rev. 128, 2425 (1962).

[57] R. Jackiw and K. Johnson, Phys. Rev. D 8, 2386 (1973).

[58] J. Smit, Phys. Rev. D 10, 2473 (1974).

[59] E. Eichten and F. Feinberg, Phys. Rev. D 10, 3254 (1974).

[60] E. C. Poggio, E. Tomboulis, and S. H. H. Tye, Phys. Rev. D 11, 2839 (1975).

[61] A. Athenodorou, D. Binosi, P. Boucaud, F. De Soto, J. Papavassiliou, J. Rodriguez-Quintero, and S. Zafeiropoulos, Phys. Lett. B 761, 444 (2016).

[62] P. Boucaud, F. De Soto, J. Rodríguez-Quintero, and S. Zafeiropoulos, Phys. Rev. D 95, 114503 (2017).

[63] P. Boucaud, F. De Soto, J. Rodríguez-Quintero, and S. Zafeiropoulos, Phys. Rev. D 96, 098501 (2017).

[64] D. Binosi and J. Papavassiliou, Phys. Rev. D 66, 025024 (2002).

[65] P. A. Grassi, T. Hurth, and A. Quadri, Phys. Rev. D 70, 105014 (2004).

[66] A. C. Aguilar and J. Papavassiliou, Phys. Rev. D 81, 034003 (2010).

[67] J. S. Ball and T.-W. Chiu, Phys. Rev. D 22, 2550 (1980).

[68] D. Binosi and J. Papavassiliou, J. High Energy Phys. 03 (2011) 121.

[69] A. Cucchieri, A. Maas, and T. Mendes, Phys. Rev. D 74, 014503 (2006).

[70] A. Cucchieri, A. Maas, and T. Mendes, Phys. Rev. D 77, 094510 (2008).

[71] R. Alkofer, M. Q. Huber, and K. Schwenzer, Eur. Phys. J. C 62, 761 (2009).

[72] M. Tissier and N. Wschebor, Phys. Rev. D 84, 045018 (2011).

[73] M. Pelaez, M. Tissier, and N. Wschebor, Phys. Rev. D 88, 125003 (2013). 
[74] A. C. Aguilar, D. Binosi, D. Ibañez, and J. Papavassiliou, Phys. Rev. D 89, 085008 (2014).

[75] A. Blum, M. Q. Huber, M. Mitter, and L. von Smekal, Phys. Rev. D 89, 061703 (2014).

[76] G. Eichmann, R. Williams, R. Alkofer, and M. Vujinovic, Phys. Rev. D 89, 105014 (2014).

[77] R. Williams, C. S. Fischer, and W. Heupel, Phys. Rev. D 93, 034026 (2016).

[78] A. G. Duarte, O. Oliveira, and P. J. Silva, Phys. Rev. D 94, 074502 (2016).

[79] J. C. Bloch, Ph.D. thesis, University of Durham, 1995.

[80] J. C. Bloch, Few-Body Syst. 33, 111 (2003).

[81] J. M. Cornwall, J. Papavassiliou, and D. Binosi, The Pinch Technique and its Applications to Non-Abelian
Gauge Theories (Cambridge University Press, Cambridge, England, 2010).

[82] D. Binosi, D. Ibañez, and J. Papavassiliou, Phys. Rev. D 86, 085033 (2012).

[83] A. C. Aguilar, D. Binosi, and J. Papavassiliou, Phys. Rev. D 89, 085032 (2014).

[84] P. Pascual and R. Tarrach, Lect. Notes Phys. 194, 1 (1984).

[85] Ph. Boucaud, F. de Soto, J. P. Leroy, A. Le Yaouanc, J. Micheli, H. Moutarde, O. Pène, and J. Rodríguez-Quintero, Phys. Rev. D 74, 034505 (2006).

[86] D. Binosi, D. Ibañez, and J. Papavassiliou, J. High Energy Phys. 09 (2014) 059.

[87] A. K. Cyrol, M. Q. Huber, and L. von Smekal, Eur. Phys. J. C 75, 102 (2015). 